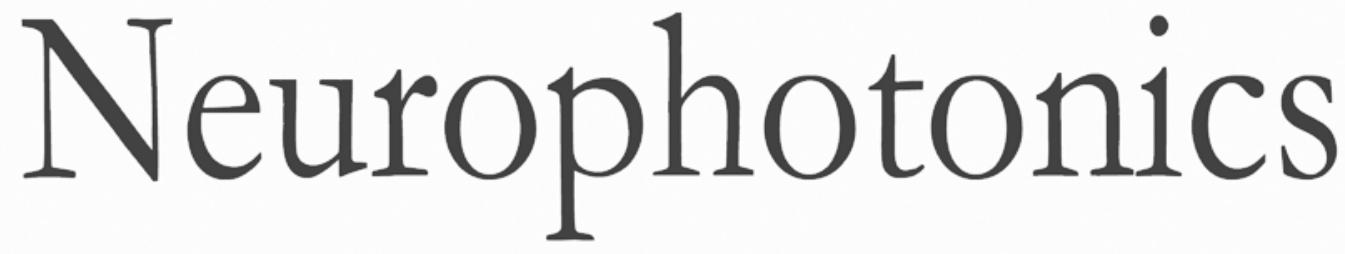

\title{
Linking brain activation to topological organization in the frontal lobe as a synergistic indicator to characterize the difference between various cognitive processes of executive functions
}

Zhishan $\mathrm{Hu}$

Juan Zhang

Lingyan Zhang

Yu-Tao Xiang

Zhen Yuan 


\title{
Linking brain activation to topological organization in the frontal lobe as a synergistic indicator to characterize the difference between various cognitive processes of executive functions
}

\author{
Zhishan Hu, ${ }^{\mathrm{a}}$ Juan Zhang, ${ }^{\mathrm{b}}$ Lingyan Zhang, ${ }^{\mathrm{C}}$ Yu-Tao Xiang, ${ }^{\mathrm{a}}$ and Zhen Yuan ${ }^{\mathrm{a}, *}$ \\ anniversity of Macau, Faculty of Health Sciences, Macao Special Administrative Region, China \\ bUniversity of Macau, Faculty of Education, Macao Special Administrative Region, China \\ 'The Third Affiliated Hospital of China Southern Medical University, Department of Radiology, Guangzhou, China
}

\begin{abstract}
Executive functions (EFs) associated with the frontal lobe are vital for goal-orientated behavior. To date, limited efforts have been made to examine the relationships among the behavior, brain activation, and topological organization of functional networks in the frontal lobe underlying various EF tasks, including inhibition, working memory, and cognitive flexibility. In this study, functional near-infrared spectroscopy neuroimaging technique was used to systematically inspect the differences in the brain activation and the topological organization of brain networks between various EF tasks in the frontal lobe. In addition, the relationships between brain activation/network properties and task performances and the relationships between brain activation and network properties were, respectively, examined for different EF tasks. Consequently, we have discovered that the nodal and global properties of the resting-state and task-evoked networks, respectively, exhibited significant correlations with the activation of various brain regions during various EF tasks. In particular, the measure that links the neural activation to the topological organization of the brain networks in the frontal lobe can serve as a synergistic indicator to examine the difference between various EF tasks, which paves a way toward a comprehensive understanding of the neural mechanism underlying EFS. ( ) The Authors. Published by SPIE under a Creative Commons Attribution 4.0 Unported License. Distribution or reproduction of this work in whole or in part requires full attribution of the original publication, including its DOI. [DOI: 10.1117/1.NPh.6.2.025008]
\end{abstract}

Keywords: functional near-infrared spectroscopy; behavior; network; activation; executive functions; frontal cortex.

Paper 18067RR received Oct. 30, 2018; accepted for publication Mar. 28, 2019; published online May 15, 2019.

\section{Introduction}

Executive functions (EFs) refer to a set of cognitive processes that are essential for the cognitive control of behavior. In particular, EFs implicate the top-down control processes, which are also vital for physical and mental health and academic and job performances. ${ }^{1}$ EFs consist of three basic cognitive processes, such as inhibition, working memory, and cognitive flexibility, in which inhibition involves resisting dominant response and focusing on the present task, and working memory is associated with holding and operating verbal or nonverbal information in mind, whereas cognitive flexibility is related to shifting between multiple tasks and adjusting the behavior appropriately to a changing circumstance. ${ }^{2}$ It is widely recognized that EFs are regulated by the frontal lobe, ${ }^{1,3}$ whereas frontal lesions or dysfunctions can cause a wide range of cognitive deficits and mental diseases, including dyslexia, ${ }^{4}$ dyscalculia,${ }^{5}$ obsessive-compulsive disorder, ${ }^{6}$ attention-deficit hyperactivity, ${ }^{7}$ and schizophrenia. ${ }^{8}$

Interestingly, recent neuroimaging and neuroscience studies demonstrate that the different brain regions in the frontal cortex are responsible for the various cognitive processes of EFs. For example, previous reports have shown that the attentional inhibition (measured by the flanker task) elicited the activation in the supplementary motor area (SMA), the right ventrolateral prefrontal cortex, the superior frontal gyrus, and the left

*Address all correspondence to Zhen Yuan, E-mail: zhenyuan@umac.mo superior/anterior parietal lobe, ${ }^{9,10}$ whereas the response inhibition induced the activation in the right inferior frontal gyrus (IFG) and the right pre-SMA. ${ }^{11-14}$ More important, during the response inhibition task, it has been discovered that the superior part of anterior frontal cortex exhibited reduced activation as compared to that at rest. ${ }^{15,16}$ In addition, working memory is considered to be linked to the functions of the dorsolateral prefrontal cortex (DLPFC). ${ }^{17-19}$ In addition, a meta-analysis study has revealed that the lateral and medial regions of the frontopolar area (FPA) get activated during the shifting between cognitive rules. ${ }^{20}$ An additional study has also illustrated that the FPA plays an essential role in monitoring multiple goals in parallel and in switching between them. ${ }^{21}$

In addition to the study of brain activation patterns, functional connectivity (FC) analyses have become an increasingly important tool for examining EFs. ${ }^{22}$ In particular, significant progress has been made in the characterization of the human brain networks, in which graph theory-based network analysis is introduced to describe more comprehensive properties of the functional topological organization of brain networks, such as the small-worldness. ${ }^{23,24}$ More importantly, the brain FC during a task is modulated primarily by the intrinsic resting-state network architectures, and then secondarily by the task-evoked networks. ${ }^{25}$ In addition, a previous study has demonstrated that more globally integrated networks enable fast and effective performance during an $n$-back task. ${ }^{26}$ Further, a recent functional near-infrared spectroscopy (fNIRS) study associated with cognitive flexibility has examined the relationship between 
topological properties of the resting-state network in the prefrontal cortex and EF scores,${ }^{27}$ in which they have discovered that the total EF scores negatively correlated with regional network properties in the right triangular IFG, whereas the EF scores positively correlated with regional properties in the right dorsal superior frontal gyrus. In addition, Zhao et al.'s study ${ }^{27}$ illustrated that the working memory is related to the regional network properties in the right middle frontal gyrus and triangular IFG, whereas the cognitive flexibility is associated with the regional properties in the right middle frontal gyrus.

Although the brain activation and FC were, respectively, examined in a single study, ${ }^{28-31}$ the relationship between these two perspectives has not been extensively explored. For example, Mennes et al. ${ }^{32}$ inspected the relationship between restingstate FC and brain activation by using the flanker task, in which they discovered that the resting-state FC within default mode networks showed a negative correlation with the task-evoked brain activation, whereas the task-based FC positively correlated with the brain activation. An additional study based on the visual attention tasks also demonstrated that the local FC density, which mainly decreased in weakly activated/deactivated brain regions, was associated with better behavioral performance and activation in the task-related networks. ${ }^{33}$

However, to the best of our knowledge, no systematic study has been performed to examine the relationship between the brain activation and the topological properties of FC during the EF tasks. As a result, it is hypothesized for the present study that the linking between the brain activation and the task-based/ resting-state FC can serve as a neural index, which might identify the significant difference between various cognitive processes of EFs, including inhibition, working memory, and cognitive flexibility.

To test this hypothesis, the brain activation and resting-state-/ task-based FC are accessed based on four classic EF tasks (go/ no-go, flank, $n$-back, and switch). The cortical hemodynamic changes at rest are first acquired and then task-based recordings are also performed for the investigation of EFs with fNIRS. The fNIRS is an emerging noninvasive optical neuroimaging technique, which can offer unsurpassed temporal resolution (50 Hz for the present system) and characterize the hemodynamic fluctuations more precisely. In addition, fNIRS can be operated in a more comfortable, portable, quiet, and natural way with very few body constraints and low cost. ${ }^{34-37}$ In particular, the brain activation and FC, in this study, are first individually explored and compared among various cognitive processes of EFs. Further, we also carefully examine how the activation and FC network are individually associated with the behavior performances of four various EF tasks. More important, we inspect the relationship between the activation and the topological properties of brain functional networks during tasks or at rest. As the linking between the brain activation and the network properties of FC has never been systematically explored for cognitive processes of EFs, this fNIRS work will pave a new avenue toward a better understanding of the cognitive mechanism of EFs.

\section{Methods and Materials}

\subsection{Participants}

A total of 30 college students (15 females and 15 males) were recruited from the campus of the University of Macau, China. Participants aged from 18 to 28 years (mean age: 23.2 years) had normal or corrected-to-normal vision without histories of learning disabilities, medical illness, neurological, or psychiatric disorders. Informed consent documents were signed prior to the experiment. This study was approved by the Medical Ethics Committee with the University of Macau.

\subsection{Procedures and Tasks}

The experimental tests were performed in a quiet room. 11-min resting-state data were acquired before the performance of $\mathrm{EF}$ tasks, which were utilized to construct the intrinsic functional networks in the frontal lobe. During the resting-state recordings, participants were instructed to close their eyes, remain still, and avoid falling asleep. Subsequently, participants went through four typical EF tasks related to different EFs components, with a short break between any two tasks with the time period determined by participants. These four tasks consisted of the flanker task, go/no-go task, $n$-back task, and switch task. In particular, there were two conditions for each of the four tasks, in which the incongruent, no-go, 2-back, and switch condition, respectively, were the experimental conditions, whereas the congruent, go, 0-back, and repeat condition, respectively, were the control conditions. The schematic of experimental procedures of the four tasks is illustrated in Fig. 1.

For the present experimental design, participants performed the flanker task first after 11-min resting-state recordings, followed by the go/no-go task. In contrast, the switch and $n$ back tasks were arranged as the last sections for data acquisition in order to reduce the possible influence of difficulty in performing the tasks. However, the order of the switch and $n$-back tasks was not well counterbalanced, whose effect should be further investigated in the future.

\subsubsection{Flanker task}

The flanker task was adopted to inspect the inhibition at the attention level. ${ }^{38,39}$ The event-related stimuli were presented trial by trial. Each trial lasted $15 \mathrm{~s}$, which consisted of a 0.5 $\mathrm{s}$ stimulus period, a 1.5 -s presentation of a blank screen, and a 13 -s rest period with a fixation cross displayed in the center of the monitor. The flank task had two congruent stimuli including " $<<<<<$ " and " $>>>>>$," and two incongruent stimuli including " $<<><<$ " and " $>><>>$." When the central arrow points to the left (" $<$ "), the participants were required to press the left button, whereas they had to press the right button if it points to the right (">"). Stimuli of each type were randomly repeated 24 times.

\subsubsection{Go/no-go task}

The go/no-go task was utilized to study the inhibition at the response level. ${ }^{40,41}$ The procedure of go/no-go task was very similar to the event-related flanker task, in which a series of English letters were presented. Participants were required to press the space key with their right forefinger as soon as the letter appeared (go condition), except the letter " $\mathrm{X}$ " (no-go condition), which needed the participants to withhold their response.

\subsubsection{N-back task}

The $n$-back task was used to investigate the working memory. ${ }^{17,42,43}$ A block-design paradigm was adopted, which contained six 0-back blocks and six 2-back blocks. Each block 


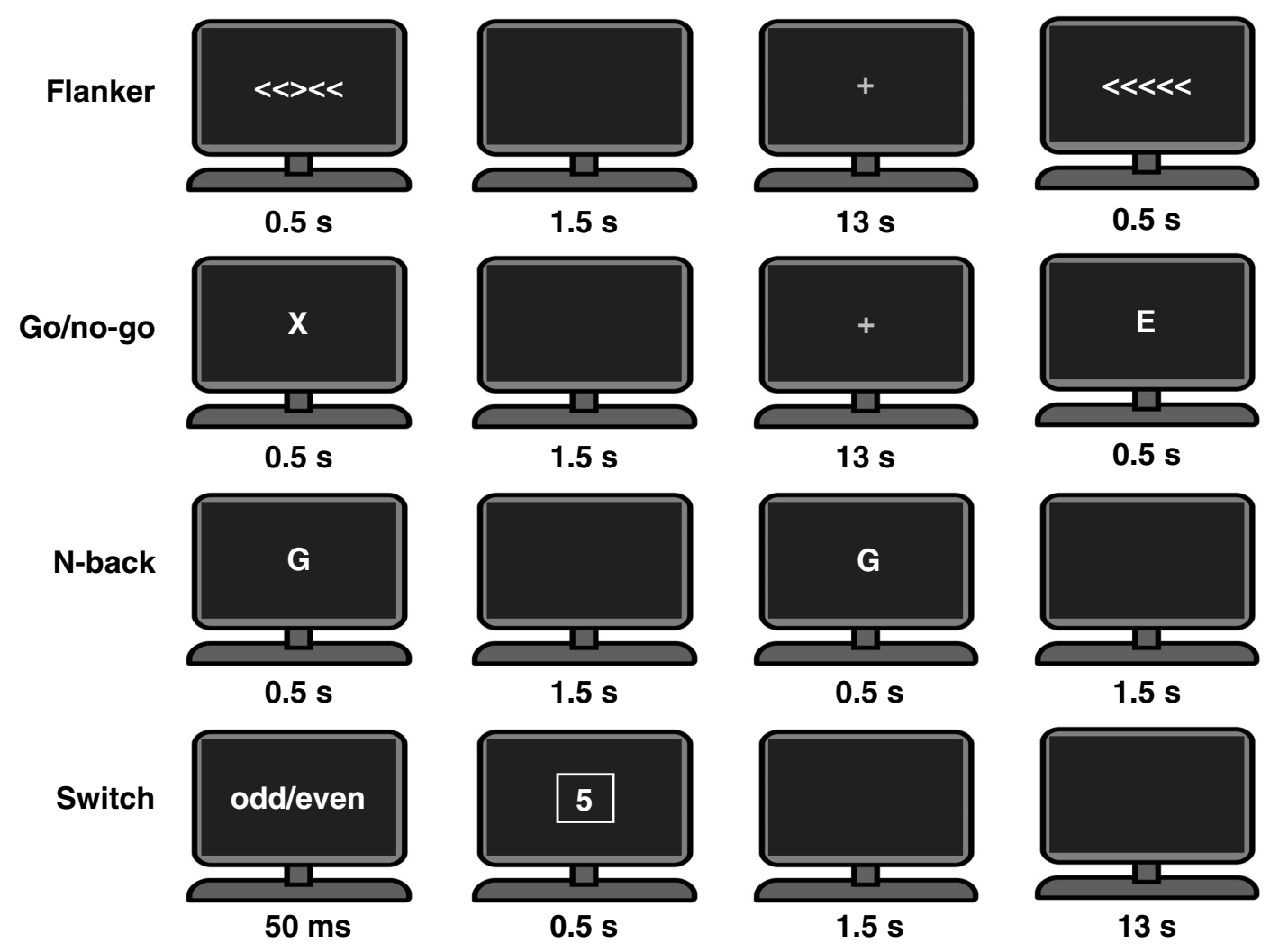

Fig. 1 A schematic representation of the experimental procedures of the four EF tasks.

had 12 trials, and each trial started with a stimulus period of $0.5 \mathrm{~s}$ with a letter in the center of the monitor, followed by a 1.5 -s blank screen (interstimulus interval). Participants need to respond to the stimuli by pressing a key with their forefinger. The stimuli materials consisted of a bunch of English letters, which were presented randomly. During the 0-back condition, participants responded to the letter "A" (target), whereas during the 2-back condition, participants needed to respond to any letter that was identical to the one presented two trials before (target). During the $n$-back task, participants were required go through 0 back and 2-back blocks in turns, and between the predecessor and the successor blocks, there was a 30-s rest period.

\subsubsection{Switch task}

The switch task was used to inspect cognitive flexibility. The procedure of switch task was quite similar to that of the flanker task, except for an additional 50-ms cue presented before the onset of the stimulus, which demonstrated the stimulus conditions (odd/even or odd/even). The stimuli materials consisted of a serial of Arabic numbers ranged from 0 to 9 . For an individual trial, a single letter was presented with a background shaped either like a square (odd/even condition) or like a diamond (low/high condition). Participants were instructed to determine whether the number was odd/even or low/high with a left or right key-press. It should be noted that in the low/high condition, the number 0 to 4 was classified as "low," whereas 5 to 9 was classified as "high." The trials were presented randomly either as switch trials or as repeat trials. In switch trials, the task was switched from the former one, whereas in repeat trials, the task was the same as the former one.

\subsection{Functional Near-Infrared Spectroscopy Data Acquisition and Preprocessing}

The fNIRS signals were acquired at a sample rate of $50 \mathrm{~Hz}$ by using a continuous wave (CW) system (CW6 fNIRS system; TechEn Inc., Milford, Massachusetts). The optode arrays comprised 8 laser sources at wavelengths 690 and $830 \mathrm{~nm}$ and 16 optical detectors, which generated 32 measurement channels. We used a homemade plastic patch to hold the optodes with a fixed interoptode distance of $3 \mathrm{~cm}$ and then placed the patch on each participant's head to cover the frontal lobe, as displayed in Fig. 2(a). After the experiment, a three-dimensional (3-D) digitizer (PATRIOT, Polhemus, Colchester, Vermont) was used to measure the 3-D coordinates of the optodes, which were further imported to NIRS_SPM ${ }^{44}$ for spatial registration to generate the 3 -D coordinates of all channels. In addition, the distributions of the optodes and channels, mean MNI coordinates of the channels, and anatomical labels are provided in Fig. 2 and Supplementary Material in Table S1, respectively.

Before data preprocessing, the signal quality of each channel was accessed by using the signal-to-noise ratio (SNR), which was the ratio of the mean to standard deviation of the signal intensity. According to Rose criterion, ${ }^{45}$ data from participants with an SNR of $<5$ for both wavelengths were excluded for further analysis as the low SNR could undermine the corresponding correlations and network properties. ${ }^{46}$ The SNRs of bad channels from each wavelength are listed in Table S2 in the Supplementary Material for the excluded participants.

The preprocessing of fNIRS data was performed with Homer2 software: ${ }^{47}$ (1) converting the raw data to the changes in optical density, (2) removing motion artifacts by spline method, $^{48}$ (3) bandpass filtering (for filtering parameter 

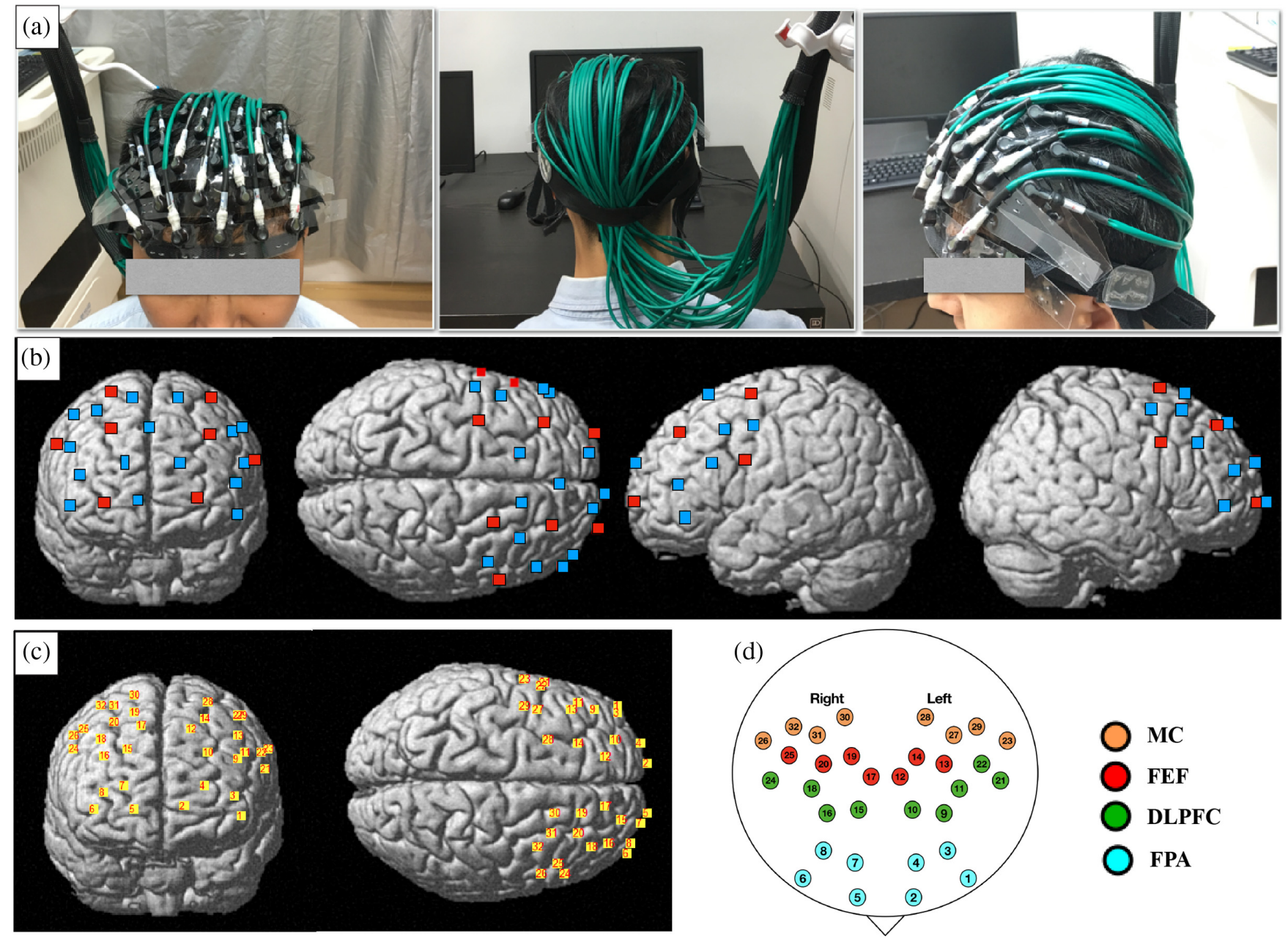

Fig. 2 The configurations of optodes and channels. (a) The front, posterior, and left view of the optodes on a participant's head. (b) The result of spatial registration of the optodes. Red squares denote the sources and blue squares denote the detectors. (c) The generated channels are labeled with numbers. (d) The anatomical labels of the channels. Dots with different colors represent different brain regions. Abbreviations: MC, premotor and supplementary motor cortex; FEF, frontal eye fields; DLPFC, dorsolateral prefrontal cortex; and FPA, frontopolar area.

selection, resting state: 0.01 to $0.08 \mathrm{~Hz} ;{ }^{49} \mathrm{EF}$ tasks: 0.01 to $0.2 \mathrm{~Hz}$ ), (4) using principal component analysis to remove the first one or two eigenvectors accounting for $80 \%$ of the variance in the optical data, which was mainly contributed by heart rate and blood pressure oscillations, ${ }^{50-53}$ (5) normalizing the concentration changes by subtracting the mean channel-wise concentration, which were further processed by dividing the standard deviation ( $z$-scores), and (6) providing baseline correction with the period from -2 to $0 \mathrm{~s}$ for a single trial. The adopted frequency band was used to filter out the high-frequency physiological noises, such as heart beat $(\sim 0.8 \mathrm{~Hz})$, cardiac cycles $(\sim 1 \mathrm{~Hz})$, and breathing $(\sim 0.2 \mathrm{~Hz}),{ }^{50,51}$ as well as the slow-frequency physiological drifts. ${ }^{54}$ In addition, for resting-state data, data from 180 to $600 \mathrm{~s}$ were extracted before the preprocessing to ensure stable signals, which were then detrended after the preprocessing. ${ }^{46}$ The method for data preprocessing was in line with those from previous studies. ${ }^{54-57}$ Importantly, both the $\mathrm{HbO}$ and $\mathrm{HbR}$ signals can be used to characterize the activation and functional network properties of the frontal lobe during the rest or EF tasks. However, most of previous fNIRS work only reported the neuroimaging results based on $\mathrm{HbO}$ signals. ${ }^{58}$
In this study, both $\mathrm{HbR}$ and $\mathrm{HbO}$ data were analyzed to generate the brain activation and construct the brain networks.

\subsection{Brain Activation}

After the preprocessing, the grand-averaged $\mathrm{HbO}$ and $\mathrm{HbR}$ data were calculated for each condition associated with each task across all participants. Then, the mean $z$-scores of $\mathrm{HbO}$ and HbR signals during the stimuli period (for the $n$-back task: $0 \mathrm{~s}$ to $26 \mathrm{~s}$; for the other EF tasks: 0 to $13 \mathrm{~s}$ ) were calculated to map the brain activation for each channel. In particular, we discovered that for most of the cases the task-related brain regions exhibited increased $\mathrm{HbO}$ and decreased $\mathrm{HbR}$ concentrations. $^{59,60}$

\subsection{Network Topological Properties}

The functional networks in frontal lobe were constructed, in which resting-state and task-evoked FC matrices were generated by computing the Pearson correlations between pairs of channels for each participant. In addition, the network topological 
properties of constructed matrices were analyzed using GRETNA. ${ }^{61}$

The network topological properties are further characterized by seven global parameters and three nodal parameters. ${ }^{62-64}$ The global parameters denote the efficiency of the whole network, which consist of the average clustering coefficient $\left(C_{p}\right)$, average characteristic path length $\left(L_{p}\right)$, normalized clustering coefficient $(\gamma)$, normalized characteristic path length $(\lambda)$, small-worldness $\sigma)$, local efficiency $\left(E_{\mathrm{loc}}\right)$, and global efficiency $\left(E_{\mathrm{glob}}\right)$. The nodal parameters represent the efficiency of nodes, which include the nodal degree $\left(K_{\text {nod }}\right)$, nodal efficiency $\left(E_{\text {nod }}\right)$, and nodal betweenness centrality $\left(\mathrm{BC}_{\text {nod }}\right)$. In brief, $C_{p}$ and $\gamma$ measure the local interconnectivity of a network, whereas $L_{p}$ and $\lambda$ quantify the overall routing efficiency of a network. In addition, $E_{\text {loc }}$ plays a role similar to the clustering coefficient, whereas $E_{\text {glob }}$ is inversely related to the characteristic path length. ${ }^{62-64}$ Further, networks with higher $\sigma$ are regarded as systems that are both locally and globally efficient. In contrast, for nodal topological properties, $K_{\text {nod }}$ denotes the number of edges linked to a node, which measures the association capability of the given node. However, $E_{\text {nod }}$ is inversely correlated with the averaged shortest path length from a given node to the other nodes, which measures the nodal efficiency of information transfer. In addition, $\mathrm{BC}_{\text {nod }}$ denotes the frequency of a given nodal showing up in a shortest path from one node to another, which exhibits the importance of a given node over the information flow. It is also noted for the present study that the threshold is determined by the network density (sparsity) of the brain networks, which is defined by the ratio $(S)$ of the existing edges divided by the maximum possible edges in the network. ${ }^{65}$ In addition, the global properties of brain networks only represent the information transmission pattern in frontal lobe, whereas the nodal properties denote the role of each channel in the information transmission associated with the frontal lobe network.

Interestingly, previous studies have shown that the restingstate brain functional networks have prominent small-world properties as well. ${ }^{27,46,66} \mathrm{In}$ this study, one sample $t$-tests against 1.1 were performed on the small-worldness of the resting-state or task-based networks constructed from $\mathrm{HbR}$ and $\mathrm{HbO}$ data, respectively. The maximum threshold $S$ was determined as $44 \%$ to ensure the prominent small-worldness of both the networks. ${ }^{67}$ In addition, the minimum threshold was determined by the average degree over all nodes $K_{\text {average }}>2 \ln (N)$ with $N=32$, denoting the number of the nodes, which can guarantee that a random graph is connected. ${ }^{64}$ The threshold range determined by this procedure was $23 \% \leq S \leq 44 \%$.

\section{Results}

\subsection{Behavioral Results}

The mean accuracy of the responses and reaction time for correct responses were, respectively, calculated for each participant. The accuracy and reaction time in each task were, respectively, subjected to paired $t$-test to reveal the performance difference between the experimental and the control conditions.

The $t$-test results are summarized in Table 1, which shows that all experimental cases consumed more reaction time than the control cases $(p<0.01)$. Meanwhile, lower accuracy is respectively detected in the flanker $(p=0.01)$, go/nono-go $(p<0.01)$, and $n$-back task (marginally significant) as compared to that from the control case.

\subsection{Brain Activation}

In addition, we compared the differences in brain activation between the experimental and the control conditions for each task.

The averaged $\mathrm{HbO} / \mathrm{HbR}$ time courses for the four EF tasks are displayed in the Supplementary Material in Figs. S1-S4. The brain $t$-map, generated from the activation difference between the experimental and the control conditions for each task, is visualized in the first two rows of Fig. 3 by using xjView toolbox $^{68}$ and BrainNet Viewer toolbox, ${ }^{69,70}$ and the detailed $t$-test results are provided in Table 2. In addition, the Pearson correlation is performed to examine the relationship between the $\mathrm{HbO}$ and the $\mathrm{HbR}$ activation, and the correlation coefficients are visualized in the third row of Fig. 3.

Figure 3 and Supplementary Material in Figs. S1-S4 show that the brain activation differences between the experimental and the control conditions exhibit different tendencies for the four tasks although only the flanker and switch tasks exhibit significant differences. Specifically, the incongruent case in flanker task induces increased $\mathrm{HbO}$ in the bilateral premotor and supplementary motor cortex (MC), whereas the switch case

Table 1 The $t$-test results of behavioral data.

\begin{tabular}{|c|c|c|c|c|c|c|c|c|}
\hline Contrast & $\mathrm{t}$ & df & $\mathrm{p}$ & $d$ & M1 & SE1 & M2 & SE2 \\
\hline flk_con_acc-flk_incon_acc & 3.58 & 29 & 0.001 & 0.92 & 0.99 & 0.01 & 0.92 & 0.02 \\
\hline gng_go_acc-gng_nogo_acc & 4.12 & 29 & $<0.001$ & 1.14 & 0.99 & $<0.01$ & 0.96 & 0.01 \\
\hline nbk_b0_acc-nbk_b2_acc & 1.92 & 29 & 0.065 & 0.47 & 0.99 & 0.01 & 0.96 & 0.01 \\
\hline swt_rep_acc-swt_SW_acc & 0.37 & 29 & 0.715 & 0.08 & 0.93 & 0.01 & 0.92 & 0.01 \\
\hline flk_con_rt-flk_incon_rt & -8.45 & 29 & $<0.001$ & -0.85 & 0.57 & 0.02 & 0.70 & 0.03 \\
\hline nbk_b0_rt-nbk_b2_rt & -6.80 & 29 & $<0.001$ & -1.61 & 0.47 & 0.01 & 0.60 & 0.02 \\
\hline swt_rep_rt-swt_sw_rt & -5.61 & 29 & $<0.001$ & -0.50 & 0.95 & 0.03 & 1.03 & 0.03 \\
\hline
\end{tabular}

Note: Abbreviations: acc, accuracy; $r t$, reaction time; $t, t$-value of the paired $t$-test; $d f$, degree of freedom; $d$, Cohen's $d ; M$, mean value, SE, standard error, 1, 2 means control and experimental cases, respectively; flk, flanker task; con, congruent; incon, incongruent; gng, go/no-go task; nbk, $n$-back task; b0, 0-back condition; b2, 2-back condition; swt, switch task; rep, repeat condition; and SW, switch condition. 


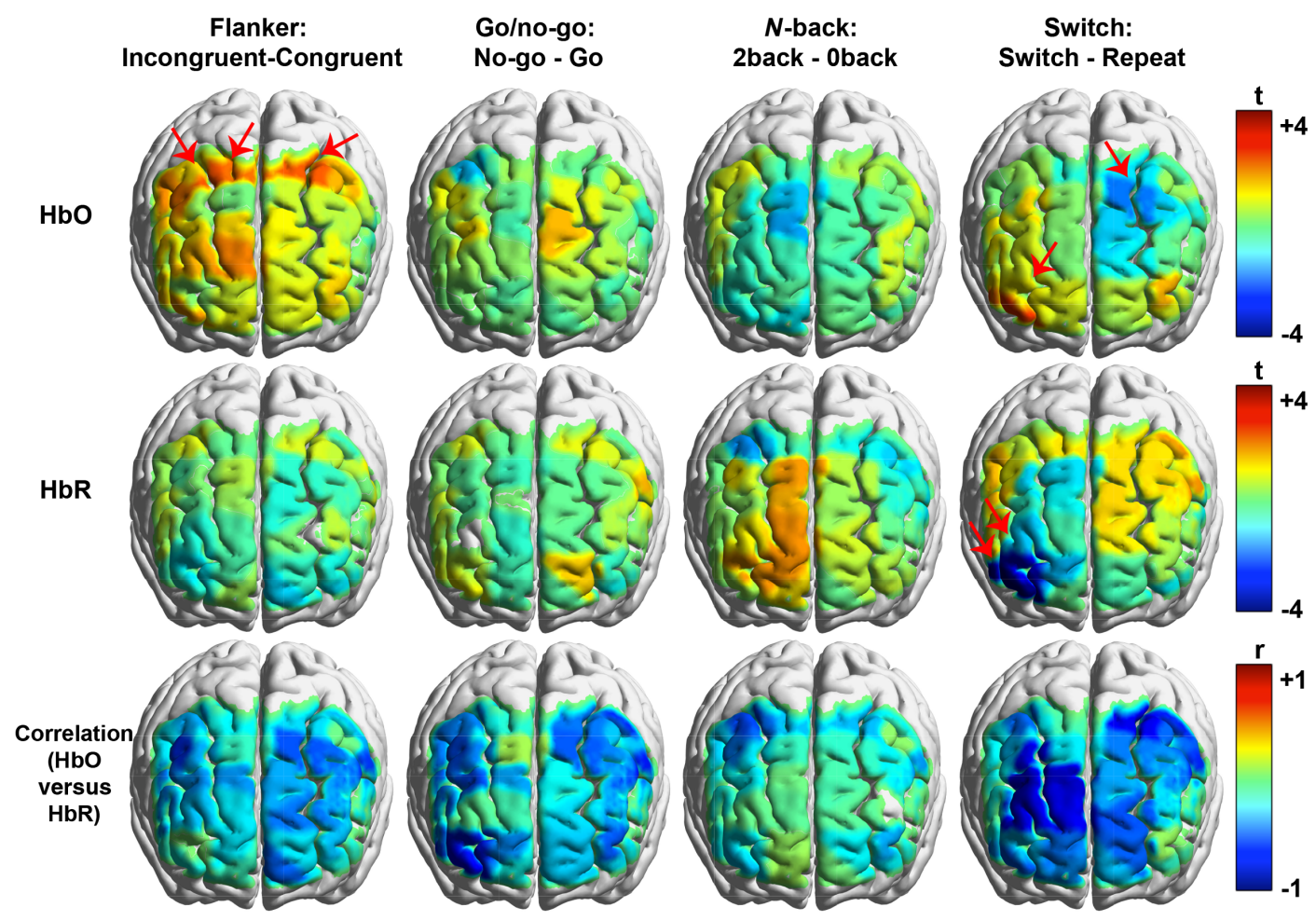

Fig. 3 Front view of the brain activation and correlation maps for the four EF tasks. Interpolated $t$-values from the contrast between the experimental and the control conditions are rendered on the brain templates in the first two rows. The bright color with positive $t$-values demonstrates that the experimental cases elicit increased $\mathrm{HbO} / \mathrm{HbR}$ activation, whereas the dark color with negative $t$-values denotes that the control cases elicit enhanced $\mathrm{HbO} / \mathrm{HbR}$ activation. In addition, the significant channels are indicated by the red arrows. In addition, correlation maps are displayed in the third row, in which the bright color denotes more positive correlation, whereas the dark color denotes more negative correlation.

Table 2 Summary of the $t$-test results with regard to the brain activation differences.

\begin{tabular}{|c|c|c|c|c|c|c|c|c|c|c|c|c|c|}
\hline & Task & \#ch & Label & $p(f d r)$ & $\mathrm{p}$ & $\mathrm{t}$ & $d f$ & SD & d & M1 & SE1 & M2 & SE2 \\
\hline \multirow[t]{5}{*}{$\mathrm{HbO}$} & Flanker (incon-con) & 28 & L-MC & 0.388 & 0.033 & 2.27 & 24 & 0.24 & 0.40 & 0.18 & 0.05 & 0.07 & 0.05 \\
\hline & & 30 & $\mathrm{R}-\mathrm{MC}$ & 0.388 & 0.048 & 2.09 & 24 & 0.31 & 0.45 & 0.22 & 0.05 & 0.09 & 0.06 \\
\hline & & 31 & $\mathrm{R}-\mathrm{MC}$ & 0.388 & 0.029 & 2.33 & 24 & 0.31 & 0.44 & 0.19 & 0.06 & 0.04 & 0.07 \\
\hline & Switch (swt-rep) & 14 & L-FEF & 0.790 & 0.049 & -2.07 & 24 & 0.22 & -0.33 & 0.07 & 0.05 & 0.16 & 0.06 \\
\hline & & 6 & R-FPA & 0.574 & 0.018 & 2.54 & 24 & 0.23 & 0.47 & 0.33 & 0.05 & 0.21 & 0.05 \\
\hline \multirow[t]{2}{*}{$\mathrm{HbR}$} & Switch (swt-rep) & 6 & R-FPA & 0.005 & $<0.001$ & -4.50 & 24 & 0.22 & -0.84 & -0.21 & 0.04 & -0.01 & 0.05 \\
\hline & & 8 & R-FPA & 0.077 & 0.005 & -3.10 & 24 & 0.20 & -0.53 & -0.07 & 0.04 & 0.05 & 0.05 \\
\hline
\end{tabular}

Note: Abbreviations: \#Ch, the label of the channel; incon, incongruent; con, congruent; swt, switch; rep, repeat; $L$, left hemisphere; $R$, right hemisphere; $t, t$-value of the paired $t$-test; $d f$, degree of freedom; $d$, Cohen's $d ; M$, mean value; SE, standard error (1,2 means the experimental and control cases, respectively).

in the switch task elicits increased $\mathrm{HbO}$ and decreased $\mathrm{HbR}$ in the right FPA and decreased $\mathrm{HbO}$ in the left frontal eye fields (FEFs). The decreased HbR is still observed even after the false discovery rate (FDR) correction. In addition, although no significant difference between the two conditions is revealed for the go/no-go and $n$-back tasks, tendencies toward enhanced
$\mathrm{HbO}$ activation for the 2-back case are observed across the task period in Supplementary Material in Fig. S3 as compared to that from the 0-back in left DLPFC (channels 9, 11, and 21). In particular, Supplementary Material in Fig. S2 shows that the no-go case elicits $\mathrm{HbR} / \mathrm{HbO}$ activation for most channels in the frontal lobe, which is also comparable to the go case. 
In addition, the negative correlations between $\mathrm{HbO}$ and $\mathrm{HbR}$ activations are clearly identified by the widespread dark color in the correlation maps in Fig. 3.

\subsection{Functional Network Properties}

\subsubsection{Functional connectivity}

The grand-averaged correlation coefficients for each condition of the four tasks and for the resting state are visualized in the matrix form in Supplementary Material in Figs. S5 and S6 for the $\mathrm{HbO}$ and the $\mathrm{HbR}$ measures, respectively. In addition, the global and nodal topological properties of networks are calculated and compared for various test cases.

\subsubsection{Small-world properties}

Whether the task-based or resting-state networks exhibited small-world property (namely $\sigma>1$ ) is inspected in this section. One sample $t$-tests are performed on the small-worldness for the five individual test cases with selected thresholds $(23 \% \leq S \leq 44 \%)$. Figure 4 shows that the brain functional networks during all test cases exhibit the prominent small-world property.

\subsubsection{Global properties}

Subsequently, we calculated the area under property curves (AUC) over the predetermined thresholds for each test case and compared the AUC between the networks during the tasks and at rest, and also between the experimental and the control conditions for the four tasks. In addition, paired $t$-tests were performed on all topological properties, including the global and nodal properties.

First, the global properties of the networks for each individual test case are compared to those at rest, and the comparison results are provided in Table 3. Interestingly, Table 3 shows that the global properties of HbR-based networks are more sensitive to the task tests as compared to those from the HbO-based ones. For example, only the 2-back case in $n$-back task exhibits decreased $C_{p}$ and $E_{\mathrm{loc}}$ for HbO-based networks when compared to those at rest. However, this is not the case for HbR-based networks, in which all task-based test cases exhibit decreased local interconnection including decreased $C_{p}, \gamma$, and $E_{\text {loc }}$. In addition, the incongruent case in flanker task also exhibits increased overall routing efficiency including decreased $L_{p}$ and $\lambda$, and increased $E_{\mathrm{glob}}$ as compared to the rest. In contrast, both cases in the go/no-go task and experimental cases in then-back and switch tasks exhibit decreased small-worldness $(\sigma)$.

In addition, we also examined the differences in global properties of the networks between the experimental and the control conditions associated with the four tasks. Interestingly, we discovered that only HbR-based networks exhibited decreased $E_{\text {loc }}[t(24)=-2.07, p=0.05]$ for the incongruent case in flanker task.

\subsubsection{Nodal properties}

We also compared the network nodal property difference between each task-based test and those from the resting state. The $t$-values are visualized in Supplementary Material in Figs. S7 and S8, and the detailed statistical analysis results are listed in Supplementary Material in Tables S3 and S4. We discovered that HbO- and HbR-based networks exhibited significant differences in nodal properties. For example, significantly decreased $\mathrm{BC}_{\text {nod }}$ was clearly identified in the left $\mathrm{MC}$ (channel 29) for HbO-based networks for all task-based tests, although it was not that significant for the go case. In contrast, HbR-based networks showed decreased network efficiency, including the betweenness, nodal degree, and nodal efficiency in the left FEF (channels 12 or 14) for all task-based tests although it was not significant for some nodal properties associated with the flanker task.

In addition, we also compared the differences in network nodal properties between the experimental and the control conditions. The comparison results are given in Table 4. Regarding the HbO-based networks, the incongruent case from the flanker task exhibited decreased $K_{\text {nod }}$ in the right FPA, and increased $K_{\text {nod }}$ and $E_{\text {nod }}$ in the right FEF. In the $n$-back task, the 2-back case showed decreased $K_{\text {nod }}$ and $E_{\text {nod }}$ in the left MC and increased $\mathrm{BC}_{\text {nod }}$ in the right $\mathrm{FPA}$, whereas the switch case
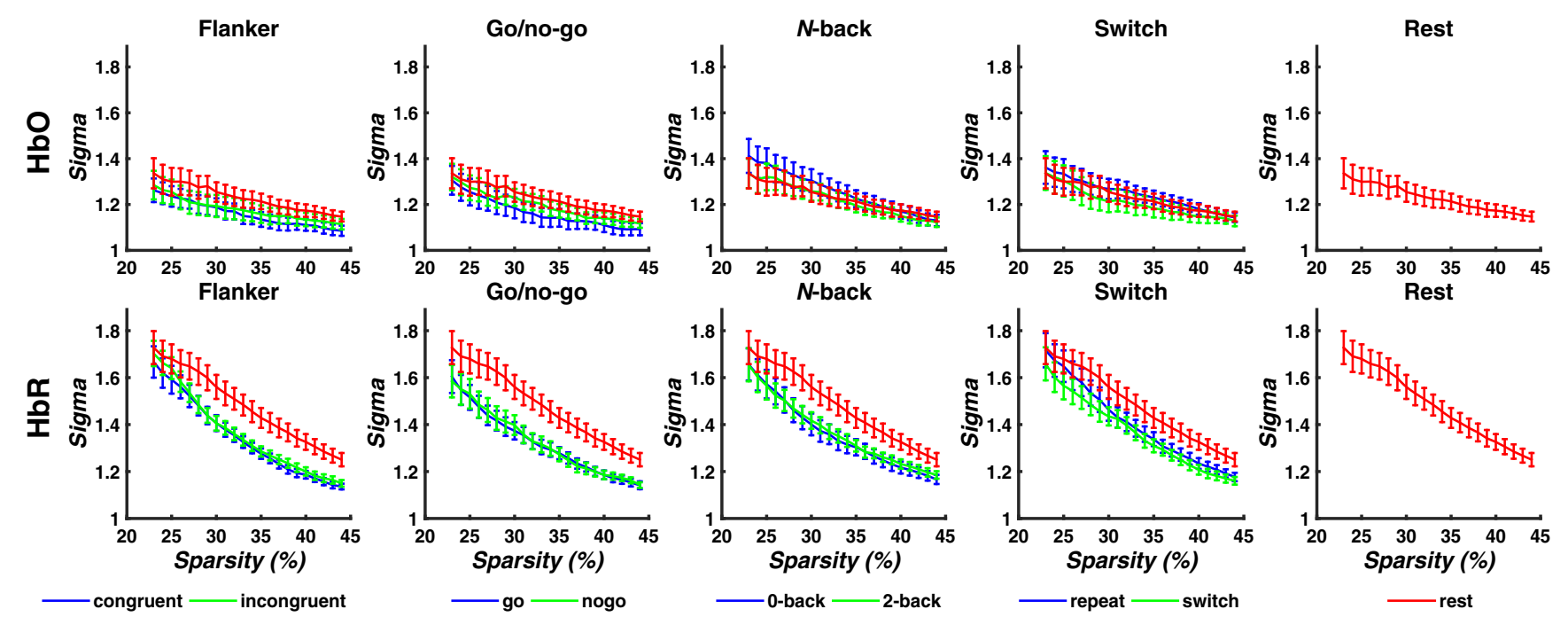

Fig. 4 Small-worldness of the brain network for each test case. Among the selected threshold (sparsity: $23 \%$ to $44 \%)$, the brain networks that exhibit the small-world property $(\sigma>1)$ are labeled with asterisks with corresponding colors. 
Table 3 Significant changes in global parameters for each condition from each task.

\begin{tabular}{|c|c|c|c|c|c|c|c|c|c|c|}
\hline & Condition & Para. & $p$ & $t$ & $d f$ & $d$ & M1 & SE1 & M2 & SE2 \\
\hline \multirow[t]{2}{*}{$\mathrm{HbO}$} & 2-Back & $\mathrm{Cp}$ & $<0.01$ & -3.17 & 24 & -0.85 & 0.64 & 0.01 & 0.68 & 0.01 \\
\hline & & Eloc & 0.02 & -2.57 & 24 & -0.72 & 0.75 & 0.01 & 0.78 & 0.01 \\
\hline \multirow[t]{22}{*}{$\mathrm{HbR}$} & Congruent & Gamma & 0.03 & -2.37 & 23 & -0.77 & 1.43 & 0.03 & 1.58 & 0.05 \\
\hline & Incongruent & $\mathrm{Cp}$ & $<0.01$ & -3.58 & 23 & -1.04 & 0.61 & 0.01 & 0.67 & 0.01 \\
\hline & & Lp & 0.02 & -2.41 & 23 & -0.70 & 1.62 & 0.02 & 1.68 & 0.02 \\
\hline & & Lambda & 0.01 & -2.80 & 23 & -0.77 & 1.04 & 0.01 & 1.07 & 0.01 \\
\hline & & Gamma & 0.02 & -2.48 & 23 & -0.77 & 1.42 & 0.03 & 1.58 & 0.05 \\
\hline & & Eloc & 0.02 & -2.55 & 23 & -0.74 & 0.76 & 0.01 & 0.79 & 0.01 \\
\hline & & Eglob & 0.02 & 2.52 & 23 & 0.73 & 0.63 & 0.01 & 0.61 & 0.01 \\
\hline & Go & Gamma & 0.01 & -2.93 & 24 & -0.87 & 1.40 & 0.03 & 1.58 & 0.05 \\
\hline & & Sigma & 0.01 & -2.80 & 24 & -0.80 & 1.32 & 0.03 & 1.47 & 0.04 \\
\hline & No-go & Gamma & 0.01 & -2.93 & 24 & -0.78 & 1.42 & 0.03 & 1.58 & 0.05 \\
\hline & & Sigma & 0.01 & -2.65 & 24 & -0.74 & 1.33 & 0.03 & 1.47 & 0.04 \\
\hline & 0-Back & $\mathrm{Cp}$ & $<0.01$ & -3.11 & 24 & -0.86 & 0.61 & 0.01 & 0.67 & 0.01 \\
\hline & & Gamma & 0.03 & -2.27 & 24 & -0.70 & 1.42 & 0.04 & 1.58 & 0.05 \\
\hline & & Eloc & 0.01 & -2.99 & 24 & -0.86 & 0.75 & 0.01 & 0.79 & 0.01 \\
\hline & 2-Back & $\mathrm{Cp}$ & $<0.01$ & -3.56 & 24 & -0.88 & 0.62 & 0.01 & 0.67 & 0.01 \\
\hline & & Gamma & 0.02 & -2.54 & 24 & -0.69 & 1.44 & 0.03 & 1.58 & 0.05 \\
\hline & & Sigma & 0.05 & -2.10 & 24 & -0.54 & 1.37 & 0.03 & 1.47 & 0.04 \\
\hline & & Eloc & 0.02 & -2.56 & 24 & -0.73 & 0.76 & 0.01 & 0.79 & 0.01 \\
\hline & Repeat & $\mathrm{Cp}$ & 0.03 & -2.28 & 23 & -0.74 & 0.63 & 0.01 & 0.67 & 0.01 \\
\hline & & Gamma & 0.03 & -2.32 & 23 & -0.63 & 1.44 & 0.03 & 1.57 & 0.05 \\
\hline & Switch & Gamma & 0.02 & -2.54 & 23 & -0.66 & 1.44 & 0.03 & 1.57 & 0.05 \\
\hline & & Sigma & 0.04 & -2.19 & 23 & -0.59 & 1.35 & 0.03 & 1.46 & 0.04 \\
\hline
\end{tabular}

from switch task showed decreased $K_{\text {nod }}$ in the left FPA and decreased $E_{\text {nod }}$ in the left FPA and right FEF.

Further, it was observed that the HbR-based networks were more sensitive to the EF demands. In the flanker task, the incongruent case exhibited increased $\mathrm{BC}_{\text {nod }}, K_{\text {nod }}$, and $E_{\text {nod }}$ in the right $\mathrm{DLPFC}$, and decreased $\mathrm{BC}_{\text {nod }}$ in the left FPA and FEF and right $\mathrm{MC}$, decreased $K_{\text {nod }}$ in the right FPA and left FEF, and deceased $\mathrm{E}_{\text {nod }}$ in the right FPA. In the $n$-back task, the 2-back case exhibited increased $\mathrm{BC}_{\text {nod }}$ in the left DLPFC and FEF and right FPA. In addition, increased $K_{\text {nod }}$ and $E_{\text {nod }}$ were identified in the right DLPFC, whereas decreased ones were revealed in the left FPA. In the switch task, we discovered that the anterior part of left DLPFC (channel 10) and its nearby regions exhibited increased $\mathrm{BC}_{\text {nod }}, K_{\text {nod }}$, and $E_{\text {nod }}$, whereas the posterior part of left DLPFC (channel 11) and its nearby regions exhibited decreased $\mathrm{BC}_{\text {nod }}, K_{\text {nod }}$, and $E_{\text {nod }}$.

\subsection{Relationship between the Brain Activation/ Network Topological Properties and Task Performance}

Subsequently, the Pearson correlation between the cognitive cost (measured by the increase in reaction time) and the brain activation/FC was also generated in this study, which can be used to examine the influence of the brain activation/networks on the task performance. As a result, the task-evoked activation and topological properties of FC were used as the measures to generate the correlations with the cognitive costs in each task. In particular, the task-evoked changes were denoted as the differences in $\mathrm{HbO}$ or $\mathrm{HbR}$ activation/topological properties between the experimental and the control conditions, whereas the cognitive cost was quantified by the relative reaction time between the experimental and the control conditions. 
Table 4 Summary of the $t$-test results with regard to the AUC of nodal parameters for each task.

\begin{tabular}{|c|c|c|c|c|c|c|c|c|c|c|c|c|c|}
\hline & Task & Para. & Label & \#Ch & $p(F D R)$ & $\mathrm{p}$ & $\mathrm{t}$ & df & $d$ & M1 & SE1 & M2 & SE2 \\
\hline \multirow[t]{9}{*}{$\mathrm{HbO}$} & Flanker (incon-con) & $\mathrm{K}$ & R-FPA & 6 & 0.76 & 0.05 & -2.09 & 24 & -0.25 & 10.35 & 1.00 & 11.60 & 0.98 \\
\hline & & $\mathrm{K}$ & R-FEF & 19 & 0.76 & 0.03 & 2.30 & 24 & 0.17 & 11.05 & 1.18 & 10.02 & 1.19 \\
\hline & & $E$ & R-FEF & 19 & 0.65 & 0.03 & 2.30 & 24 & 0.18 & 0.56 & 0.04 & 0.52 & 0.04 \\
\hline & $N$-back (b2-b0) & $\mathrm{BC}$ & R-FPA & 6 & 0.81 & 0.03 & 2.38 & 25 & 0.48 & 9.38 & 1.71 & 5.75 & 1.18 \\
\hline & & $\mathrm{K}$ & L-MC & 23 & 0.57 & 0.02 & -2.47 & 25 & -0.31 & 6.57 & 1.13 & 8.41 & 1.17 \\
\hline & & $E$ & L-MC & 23 & 0.99 & 0.04 & -2.15 & 25 & -0.36 & 0.43 & 0.05 & 0.51 & 0.04 \\
\hline & Switching (swt-rep) & $\mathrm{K}$ & L-FPA & 3 & 0.66 & 0.04 & -2.20 & 24 & -0.19 & 9.86 & 1.24 & 11.01 & 1.17 \\
\hline & & $E$ & L-FPA & 3 & 0.59 & 0.04 & -2.21 & 24 & -0.16 & 0.53 & 0.05 & 0.57 & 0.04 \\
\hline & & $E$ & R-FEF & 25 & 0.43 & 0.01 & -2.67 & 24 & -0.19 & 0.52 & 0.04 & 0.56 & 0.04 \\
\hline \multirow[t]{25}{*}{$\mathrm{HbR}$} & Flanker (incon-con) & $\mathrm{BC}$ & L-FPA & 1 & 0.32 & 0.03 & -2.39 & 24 & -0.67 & 8.53 & 1.46 & 16.73 & 3.15 \\
\hline & & $\mathrm{BC}$ & L-FEF & 13 & 0.32 & 0.05 & -2.07 & 24 & -0.28 & 9.01 & 1.72 & 11.83 & 2.26 \\
\hline & & $\mathrm{BC}$ & R-DLPFC & 16 & 0.32 & 0.03 & 2.30 & 24 & 0.59 & 25.75 & 2.73 & 17.15 & 3.10 \\
\hline & & $\mathrm{BC}$ & $\mathrm{R}-\mathrm{MC}$ & 31 & 0.25 & 0.01 & -2.90 & 24 & -0.62 & 12.10 & 2.28 & 21.90 & 3.84 \\
\hline & & $\mathrm{K}$ & R-FPA & 6 & 0.29 & 0.02 & -2.53 & 24 & -0.52 & 12.37 & 0.60 & 13.91 & 0.59 \\
\hline & & $\mathrm{K}$ & L-FEF & 13 & 0.46 & 0.04 & -2.14 & 24 & -0.38 & 10.07 & 0.58 & 11.42 & 0.83 \\
\hline & & $\mathrm{K}$ & R-DLPFC & 16 & 0.13 & $<0.01$ & 3.19 & 24 & 0.66 & 14.02 & 0.57 & 11.93 & 0.70 \\
\hline & & $\mathrm{E}$ & R-FPA & 6 & 0.41 & 0.03 & -2.37 & 24 & -0.48 & 0.67 & 0.01 & 0.70 & 0.01 \\
\hline & & $E$ & R-DLPFC & 16 & 0.03 & $<0.01$ & 3.78 & 24 & 0.76 & 0.70 & 0.01 & 0.65 & 0.02 \\
\hline & $N$-back (b2-b0) & $\mathrm{BC}$ & R-FPA & 6 & 0.16 & 0.01 & 2.78 & 25 & 0.81 & 21.47 & 3.20 & 10.62 & 1.87 \\
\hline & & $\mathrm{BC}$ & L-DLFPC & 10 & 0.41 & 0.05 & 2.10 & 25 & 0.54 & 19.94 & 2.76 & 13.49 & 1.78 \\
\hline & & $\mathrm{BC}$ & L-FEF & 14 & 0.16 & 0.01 & 2.79 & 25 & 0.74 & 18.69 & 3.06 & 9.59 & 1.54 \\
\hline & & $\mathrm{K}$ & L-FPA & 1 & 0.08 & $<0.01$ & -3.36 & 25 & -0.65 & 10.82 & 0.91 & 13.51 & 0.71 \\
\hline & & $\mathrm{K}$ & R-DLPFC & 24 & 0.62 & 0.04 & 2.18 & 25 & 0.51 & 12.71 & 0.63 & 10.89 & 0.78 \\
\hline & & $E$ & L-FPA & 1 & 0.14 & $<0.01$ & -3.15 & 25 & -0.69 & 0.63 & 0.02 & 0.69 & 0.01 \\
\hline & & $E$ & R-DLPFC & 24 & 0.57 & 0.05 & 2.10 & 25 & 0.49 & 0.67 & 0.01 & 0.64 & 0.01 \\
\hline & Switching (swt-rep) & $\mathrm{BC}$ & L-DLFPC & 10 & 0.43 & 0.03 & 2.25 & 24 & 0.35 & 17.25 & 2.04 & 13.32 & 2.48 \\
\hline & & $\mathrm{BC}$ & L-DLFPC & 11 & 0.43 & 0.03 & -2.38 & 24 & -0.44 & 9.70 & 1.84 & 14.38 & 2.38 \\
\hline & & $\mathrm{BC}$ & L-MC & 23 & 0.43 & 0.04 & -2.12 & 24 & -0.50 & 8.19 & 1.33 & 13.16 & 2.48 \\
\hline & & $\mathrm{K}$ & L-DLFPC & 10 & 0.53 & 0.05 & 2.07 & 24 & 0.49 & 13.88 & 0.58 & 12.36 & 0.66 \\
\hline & & $\mathrm{K}$ & L-DLFPC & 11 & 0.53 & 0.03 & -2.36 & 24 & -0.30 & 9.70 & 0.85 & 10.96 & 0.81 \\
\hline & & $\mathrm{K}$ & R-FEF & 17 & 0.53 & 0.04 & 2.18 & 24 & 0.45 & 8.99 & 0.79 & 7.50 & 0.51 \\
\hline & & $E$ & L-DLFPC & 10 & 0.49 & 0.04 & 2.17 & 24 & 0.50 & 0.70 & 0.01 & 0.67 & 0.01 \\
\hline & & $E$ & L-DLFPC & 11 & 0.10 & $<0.01$ & -3.30 & 24 & -0.31 & 0.61 & 0.02 & 0.64 & 0.02 \\
\hline & & E & L-FEF & 13 & 0.49 & 0.05 & -2.11 & 24 & -0.37 & 0.62 & 0.01 & 0.65 & 0.02 \\
\hline
\end{tabular}


Note that the go/no-go task was excluded from this analysis because no reaction time was measured in no-go condition. We discovered that only $\mathrm{HbR}$ activation (but not $\mathrm{HbO}$ ) exhibited correlations with the cognitive cost. In particular, in the flanker task, the cognitive cost showed positive correlation with HbR changes in the left FEF, whereas it showed negative correlation in the right FPA. In the $n$-back and switch tasks, positive correlations were revealed in the right DLPFC. However, after the FDR correction, no significant correlation was identified.

In contrast, for both HbO-based and HbR-based networks, the nodal (but not global) topological properties changes showed significant relationships with the cognitive cost. For example, for HbO-based networks, the cognitive cost was positively correlated with $K_{\text {nod }}$ in the left FPA and DLPFC, and positively correlated with $E_{\text {nod }}$ in the left DLPFC for flanker task, although it showed negative correlation with $\mathrm{BC}_{\text {nod }}$ in the left DLPFC and MC. Interestingly, in the $n$-back task, negative correlations were identified for $K_{\text {nod }}$ in the left FEF and for $\mathrm{BC}_{\text {nod }}$ in the left DLPFC. However, in the switch task, the cognitive cost was positively correlated with $K_{\text {nod }}$ in the right FEF, whereas negative correlations were revealed for $\mathrm{K}_{\text {nod }}$ in the right DLPFC, for $E_{\text {nod }}$ in the left MC, and for $\mathrm{BC}_{\text {nod }}$ in the left FEF and $\mathrm{MC}$ and right FPA. In contrast, after the FDR correction, only negative correlation for $\mathrm{BC}_{\text {nod }}$ was identified in the left DLPFC for flanker task.

In addition, for HbR-based networks, the cognitive cost showed negative correlation with $\mathrm{BC}_{\text {nod }}$ in the left DLPFC and right $\mathrm{MC}$ for flanker task. In the $n$-back task, positive correlations were discovered between $K_{\text {nod }}$ and cognitive cost in right $\mathrm{MC}$ and FEF and negative ones for $E_{\text {nod }}$ in the bilateral DLPFC. In the switch task, positive correlations were identified for $K_{\text {nod }}$ and $E_{\text {nod }}$ in the left FPA, whereas negative correlations were identified for $K_{\text {nod }}$ and $E_{\text {nod }}$ in the left $\mathrm{MC}$, and for $\mathrm{BC}_{\text {nod }}$ in the left DLPFC and right MC. However, this is not the case after the FDR correction, in which no significant correlation was identified.

\subsection{Relationship between Brain Activation and Networks Topological Properties}

Pearson correlation analysis was performed to examine the relationship between the brain activation and the networks topological properties. In particular, we correlated the topological properties of resting-state networks, and task-based network topological properties with task-based brain activation.

Interestingly, the brain activation during the EF tasks exhibited significant correlations with the network topological properties for most of the channels. However, after the FDR correction, it was clearly observed that the brain activation during flanker, $n$-back, and switch tasks only showed correlation with the intrinsic network properties. In contrast, in the go/no-go task, the activation not only exhibited the correlation with the topological properties derived from intrinsic networks but also exhibited the properties derived from task-evoked and HbRbased networks.

In particular, in the flanker task, the activation in bilateral DLPFC and left FEF showed negative correlation with $\lambda$ for HbO-based intrinsic networks, whereas the activation in left MC was positively correlated with $K_{\text {nod }}$ for the same restingstate networks.

In the $n$-back task, the activation in right FEF was positively correlated with $K_{\text {nod }}$ for HbO-based intrinsic networks. In addition, the activation in right $\mathrm{MC}$ was positively correlated with
$\mathrm{BC}_{\text {nod }}$ for HbR-based intrinsic networks, whereas the activation in left $\mathrm{MC}$ was negatively correlated with $\mathrm{BC}_{\text {nod }}$ from the same network. In the switch task, the activation in left FPA was negatively correlated with $C_{p}, L_{p}$, and $\lambda$, whereas it was positively correlated with $E_{\text {glob }}$ in HbR-based intrinsic networks.

In the go/no-go task, the activation in left FEF was positively correlated with $K_{\text {nod }}$ and $E_{\text {nod }}$ for HbO-based and task-evoked networks. In addition, the activation in left $\mathrm{MC}$ was negatively correlated with $C_{p}$ and $E_{\text {loc }}$ for HbR-based and task-evoked networks. Moreover, the activation in right FEF was positively correlated with $\gamma$ for the HbR-based intrinsic networks, whereas the activation in right FEF and DLPFC was positively correlated with $\sigma$ from the same networks.

\section{Discussion}

To the best of our knowledge, this is the first study that systematically explored the differences in frontal $\mathrm{HbO} / \mathrm{HbR}$-based activation and networks topological properties between various $\mathrm{EF}$ components by testing the same group of participants with fNIRS. In particular, we inspected the relationship between the activation/networks topological properties and the task performance. In addition, the relationship between the brain activation and the networks topological properties was also carefully examined as a synergistic characteristic, which can serve as an index to inspect the difference between various EFs tasks in frontal cortex.

\subsection{Differences in Behavioral Performance and Brain Activation/Networks Topological Properties}

Consistent with previous studies, ${ }^{1,71}$ the present behavioral results showed that the experimental condition consumed more cognitive effort than the control condition, which was demonstrated by both the low accuracy and the slow response for the experimental condition.

In addition, the negative relationship between $\mathrm{HbO}$ and $\mathrm{HbR}$ activation was well documented in previous studies, ${ }^{59,60}$ which was also confirmed in the present study, as indicated by the correlation maps in Fig. 3. Moreover, different activation tendencies were identified for the four EF tasks, indicating that the subcomponents of EFs were associated with different neural substrates. Specifically, the attentional inhibition elicited enhanced activation in MC. In contrast, the no-go case elicited comparable activation with the go case in frontal lobe, which confirmed the hypothesis that the response inhibition was a facet of goal-oriented response selection rather than passive inhibition. ${ }^{11,72}$ In particular, the working memory was associated with the activation in left DLPFC, whereas the cognitive flexibility was correlated more with the activation in right FPA.

More important, the network properties provided more comprehensive information to characterize the differences between various EF cognitive processes. In particular, the topological properties of HbO-based networks showed the differences from those from HbR-based networks. It was discovered that the HbR-based networks were highly clustered in the frontal lobe as compared to HbO-based ones. Moreover, both the HbO-based and HbR-based networks exhibited prominent small-world properties whether at test or during task.

For global properties of HbR-based networks, the taskevoked networks exhibited predominantly decreased network efficiency as compared to the intrinsic networks, except that of the incongruent case in the flanker task, exhibiting increased 
global efficiency. However, for HbO-based networks, only the 2-back case in $n$-back task exhibited decreased local efficiency. The new results indicated that HbR-based network properties were better indexed to characterize the network difference between various EF components. In addition, decreased network efficiency demonstrated that different brain regions worked independently to support the goal-oriented behaviors, which was also confirmed by the highly clustered FC displayed in the matrix of correlation coefficients in Supplementary Material in Fig. S6. Interestingly, unlike other EF tasks, only the incongruent case in flanker task exhibited decreased local efficiency as compared to the control condition. In addition, only the incongruent case exhibited increased global efficiency, which indicated higher routing efficiency. Our findings also suggested that the attentional inhibition was achieved in a more integrative way.

Regarding nodal properties, both the HbO-based and the HbR-based networks exhibited decreased network efficiency during EF tasks, as compared to those at rest. However, in the HbO-based networks, the left MC decreased its importance over the information flow, whereas in the HbR networks, the left FEF exhibited decreased network efficiency for all three nodal properties. Consistent with the global properties results, the nodal property analysis revealed the reorganization of the functional networks during EF tasks, in which HbO-based and HbRbased networks exhibited decreased network efficiency in different brain regions.

Importantly, when the nodal property differences were inspected between the experimental and the control conditions, multiple regions exhibited the significance, which demonstrated that the nodal properties were better indicators than the activation to characterize the neural basis-supporting EF tasks. In particular, HbO-based networks exhibited increased network efficiency including "importance over the information flow $\left(\mathrm{BC}_{\text {nod }}\right)$," "association capability $\left(\mathrm{K}_{\mathrm{nod}}\right)$," and "efficiency of the information transfer $\left(E_{\text {nod }}\right)$ " in the right DLPFC, whereas they exhibited decreased network efficiency in FPA, MC, and FEF during flanker task. The results revealed that the right DLPFC, although not significantly activated, played an important role in the organization of HbO-based networks supporting the attentional inhibition. In addition, in the $n$-back task, the right FPA played an essential role over the information flow during the 2-back case. Moreover, the left FPA exhibited decreased association capability and information transfer efficiency for the switch case in switch task.

Likewise, nodal differences in multiple channels between the experimental and the control conditions were also identified for HbR-based networks, which were in line with those of the HbObased networks. In particular, similar to HbO-based networks, HbR-based networks exhibited increased network efficiency in the right DLPFC during flanker tasks. This finding confirmed the important role of the right DLPFC during attentional inhibition. Moreover, the $n$-back task also exhibited increased importance in the left DLPFC and FEF associated with HbR-based networks, in addition to the right FPA. These results were consistent with the previous studies emphasizing the role of left DLPFC in working memory. ${ }^{73,74}$ In the switch task, decreased nodal degree and efficiency were also observed in the left FPA and right FEF although no significant difference was identified between the two conditions. Importantly, in addition to the activation of DLPFC associated with cognitive flexibility, ${ }^{75}$ we also discovered increased network efficiency in the anterior part of left DLPFC and decreased network efficiency in the posterior part.

Importantly, for the go/no-go task, the no-go condition exhibited identical brain activation and network properties with the go case. Our findings provided strong evidence that the response inhibition was a facet of goal-oriented response selection rather than passive cognitive inhibition.

\subsection{Correlations between Network Properties/Brain Activation and Behavioral Performance}

Further, we correlated the activation and network properties with behavioral performances, which were measured by the cognitive cost. Interestingly, significant correlations were revealed between network topological properties and behavioral performances. However, for activation, only the HbR case showed correlations with the behavioral performance. For example, in the flanker task, decreased HbR activation in the left FEF was associated with fast response, whereas decreased $\mathrm{HbR}$ activation in the right FPA was related to slow response. In addition, in the $n$ back and switch tasks, decreased HbR activation in the right DLPFC was associated with faster response. However, after the FDR correction, no significant correlation was identified.

More important, we discovered that the nodal topological properties, rather than global properties, of HbO-based and HbR-based networks exhibited significant correlations with the cognitive cost, which indicated that the nodal properties were more sensitive to the cognitive cost changes. Our finding was in line with a previous study, which also showed no significant correlation between the global topological properties and the behavioral performance. ${ }^{27}$

Interestingly, although the left MC exhibited decreased HbObased network efficiency during task when compared with that at rest, its importance over the information flow enhanced the performance in the flanker task, its association capability was correlated with faster response in $n$-back task, and its efficiency of information transfer and importance over the information flow were associated with better performance in switch task. As a result, the left MC played a key role in the function of frontal networks, which promoted the behavioral performance.

In HbR-based networks, the importance of left DLPFC and right $\mathrm{MC}$ over the information flow was associated with better performance during flanker task. In addition, in the $n$-back task, the association capability in right MC and FEF inhibited the behavioral performance, whereas the information transfer efficiency in bilateral DLPFC promoted the performance. In the switch task, the association capability and nodal efficiency in left FPA were associated with poor performance, whereas these properties in the left MC promoted the performance. In addition, the importance of left DLPFC and right MC was also associated with better performance.

\subsection{Relationships between Brain Activation and Networks Topological Properties}

Finally, we generated the correlations between the activation and the networks topological properties, in which multiple correlations were identified. For example, in the flanker, $n$-back, and switch tasks, the activation was mainly modulated by the intrinsic network properties. These findings are in line with the previous reports that the brain FC during task is modulated primarily by the intrinsic resting-state network architectures, ${ }^{25}$ and the resting-state networks can predict the brain activation 
and behavioral performance associated with working memory. ${ }^{76}$ In addition, the activation during the go/no-go task was not only shaped by the intrinsic network but also regulated by the taskevoked and HbR-based networks.

In addition, in the flanker task, the overall routing efficiency of networks was associated with increased $\mathrm{HbO}$ activation in bilateral DLPFC and left FEF. In addition, the association capability in left $\mathrm{MC}$ also enhanced the $\mathrm{HbO}$ activation. In the $n$-back task, the association capability of right FEF showed the positive relationship with the $\mathrm{HbO}$ activation; the importance of the right MC showed the positive correlation with the HbR activation, whereas the importance of the left MC exhibited the negative correlation with $\mathrm{HbR}$ activation. In the switch task, the local efficiency was associated with decreased $\mathrm{HbR}$ activation in the left FPA, whereas the global efficiency was associated with increased HbR activation. In addition, for the task-evoked networks during go/no-go task, the association capability and nodal efficiency in left FEF showed positive correlation with the $\mathrm{HbO}$ activation, whereas the local efficiency decreased the HbR activation in left MC. In addition, for the intrinsic network during go/no-go task, the local efficiency enhanced the HbR activation in right FEF, whereas the small-worldness increased the HbR activation in right FEF and DLPFC.

\section{Conclusions}

A systematical investigation of brain activation and FC patterns during different EF tasks was performed. Our findings demonstrate that the fNIRS is a sufficient technique to characterize the topological organization of brain networks and its link with the activation. In particular, linking the neural activation to the topological organization in frontal lobe can serve as synergistic index to examine the difference between various EF tasks, which paves a new way toward a comprehensive understanding of neural basis underlying the EFs.

\section{Disclosures}

No conflicts of interest, financial or otherwise, are declared by the authors.

\section{Acknowledgments}

This work was supported by MYRG 2016-00110-FHS and MYRG 2018-00081-FHS from the Research Services and Knowledge Transfer Office with the University of Macau in Macau, and FDCT 0011/2018/A1 and FDCT 025/2015/A1 from the Science and Technology Development Fund with the Macao government. Z.Y. and Z.H. designed the research and wrote the paper; Z.H. performed the research and analyzed the data; J.Z., L.Z., and Y.X. joined the discussion, provided professional opinions, and proofread the manuscript.

\section{References}

1. A. E. Doyle, "Executive functions in attention-deficit/hyperactivity disorder," J. Clin. Psychiatry 67(Suppl. 8), 21-26 (2006).

2. A. Miyake et al., "The unity and diversity of executive functions and their contributions to complex 'frontal lobe' tasks: a latent variable analysis," Cogn. Psychol. 41(1), 49-100 (2000).

3. J. Ericsson et al., "Involvement of sterol carrier protein-2 in dolichol biosynthesis,” J. Biol. Chem. 266(16), 10602-10607 (1991).

4. M. A. Eckert et al., "Anatomical correlates of dyslexia: frontal and cerebellar findings," Brain 126(2), 482-494 (2003).

5. B. Butterworth, S. Varma, and D. Laurillard, "Dyscalculia: from brain to education," Science 332(6033), 1049-1053 (2011).
6. P. R. Szeszko et al., "Orbital frontal and amygdala volume reductions in obsessive-compulsive disorder," Arch. Gen. Psychiatry 56(10), 913-919 (1999).

7. R. A. Barkley, "Behavioral inhibition, sustained attention, and executive functions: Constructing a unifying theory of ADHD," Psychol. Bull. 121(1), 65-94 (1997).

8. B. S. Oken et al., "Randomized, controlled, six-month trial of yoga in healthy seniors: Effects on cognition and quality of life," Altern. Ther. Health Med. 12(1), 40-47 (2006).

9. E. Hazeltine, R. Poldrack, and J. D. E. Gabrieli, "Neural activation during response competition," J. Cogn. Neurosci. 12(Suppl. 2), 118129 (2001).

10. C. Corradi-Dell'Acqua et al., "Disentangling self- and fairness-related neural mechanisms involved in the ultimatum game: an fMRI study," Soc. Cogn. Affect. Neurosci. 8(4), 424-431 (2013).

11. A. R. Aron, T. W. Robbins, and R. A. Poldrack, "Inhibition and the right inferior frontal cortex: one decade on," Trends Cogn. Sci. 18(4), 177-185 (2014).

12. I. Obeso et al., "Stimulation of the pre-SMA influences cerebral blood flow in frontal areas involved with inhibitory control of action," Brain Stimul. 6(5), 769-776 (2013).

13. I. Obeso et al., "Theta burst magnetic stimulation over the pre-supplementary motor area improves motor inhibition," Brain Stimul. 10(5), 944-951 (2017).

14. A. Bari and T. W. Robbins, "Inhibition and impulsivity: behavioral and neural basis of response control," Prog. Neurobiol. 108, 44-79 (2013).

15. S. C. Wriessnegger et al., "The interplay of prefrontal and sensorimotor cortices during inhibitory control of learned motor behavior," Front. Neuroeng. 5(July), 1-12 (2012).

16. A. H. Rodrigo et al., "Differentiating functions of the lateral and medial prefrontal cortex in motor response inhibition," Neuroimage 85, 423431 (2014).

17. A. M. Owen et al., "N-back working memory paradigm: a meta-analysis of normative functional neuroimaging studies," Hum. Brain Mapp. 25(1), 46-59 (2005).

18. D. A. Markowitz, C. E. Curtis, and B. Pesaran, "Multiple component networks support working memory in prefrontal cortex," Proc. Natl. Acad. Sci. 112(35), 11084-11089 (2015).

19. D. E. Nee et al., "A meta-analysis of executive components of working memory," Cereb. Cortex 23(2), 264-282 (2013).

20. C. Kim et al., "Domain general and domain preferential brain regions associated with different types of task switching: a meta-analysis," Hum. Brain Mapp. 33(1), 130-142 (2012).

21. F. A. Mansouri et al., "Managing competing goals-a key role for the frontopolar cortex," Nat. Rev. Neurosci. 18(11), 645-657 (2017).

22. D. M. Halepoto, L. Y. AL-Ayadhi, and A. A. A. Salam, "Therapeutic use of hyperbaric oxygen therapy for children with autism spectrum disorder," J. Coll. Phys. Surg. Pakistan 24(7), 508-514 (2014).

23. X. Liao, A. V. Vasilakos, and Y. He, "Small-world human brain networks: perspectives and challenges," Neurosci. Biobehav. Rev. 77(March), 286-300 (2017).

24. F. S. Racz et al., "Increased prefrontal cortex connectivity during cognitive challenge assessed by fNIRS imaging," Biomed. Opt. Express 8(8), 3842 (2017).

25. M. W. Cole et al., "Intrinsic and task-evoked network architectures of the human brain," Neuron 83(1), 238-251 (2014).

26. J. M. Shine et al., "The dynamics of functional brain networks: integrated network states during cognitive task performance," Neuron 92(2), 544-554 (2016).

27. J. Zhao et al., "Linking resting-state networks in the prefrontal cortex to executive function: a functional near infrared spectroscopy study," Front. Neurosci. 10(Oct), 1-17 (2016).

28. M. Y. Wang et al., "Optical mapping of prefrontal brain connectivity and activation during emotion anticipation," Behav. Brain Res. 350(2010), 122-128 (2018).

29. Z. Hu et al., "Optical mapping of brain activation and connectivity in occipitotemporal cortex during Chinese character recognition," Brain Topogr. 0(0), 1-15 (2018).

30. J. O. Maximo et al., "Task-dependent changes in frontal-parietal activation and connectivity during visual search," Brain Connect. 6(4), 335-344 (2016). 
31. A. C. K. van Duijvenvoorde et al., "Testing a dual-systems model of adolescent brain development using resting-state connectivity analyses," Neuroimage 124, 409-420 (2016).

32. M. Mennes et al., "Inter-individual differences in resting-state functional connectivity predict task-induced BOLD activity," Neuroimage 50(4), 1690-1701 (2010).

33. D. Tomasi et al., "Functional connectivity and brain activation: a synergistic approach," Cereb. Cortex 24(10), 2619-2629 (2014).

34. Z. Yuan, "Combining independent component analysis and Granger causality to investigate brain network dynamics with fNIRS measurements," Biomed. Opt. Express 4(11), 2629 (2013).

35. Z. Yuan and J. Ye, "Fusion of fNIRS and fMRI data: identifying when and where hemodynamic signals are changing in human brains," Front. Hum. Neurosci. 7(October), 676 (2013).

36. Z. Yuan, "Combining ICA and Granger causality: a novel tool for investigation of brain dynamics and brain oscillations using fNIRS measurements," Proc. SPIE 8928, 89280I (2014).

37. H. F. Ieong and Z. Yuan, "Emotion recognition and its relation to prefrontal function and network in heroin plus nicotine dependence: a pilot study," Neurophotonics 5(02), 025011 (2018).

38. R. L. Olson et al., "Neurophysiological and behavioral correlates of cognitive control during low and moderate intensity exercise," Neuroimage 131, 171-180 (2016).

39. B. A. Eriksen and C. W. Eriksen, "Effects of noise letters upon the identification of a target letter in a nonsearch task," Percept. Psychophys. 16(1), 143-149 (1974).

40. M. Criaud and P. Boulinguez, "Have we been asking the right questions when assessing response inhibition in go/no-go tasks with fMRI? A meta-analysis and critical review," Neurosci. Biobehav. Rev. 37(1), 11-23 (2013).

41. F. Hoeft et al., "Fronto-striatal dysfunction and potential compensatory mechanisms in male adolescents with fragile X syndrome," Hum. Brain Mapp. 28(6), 543-554 (2007).

42. J. D. Cohen et al., "Temporal dynamics of brain activation during a working memory task," Nature 386(6625), 604-611 (1997).

43. T. S. Braver et al., "A parametric study of prefrontal cortex involvement in human working memory," Neuroimage 5(1), 49-62 (1997).

44. J. C. Ye et al., "NIRS-SPM: statistical parametric mapping for nearinfrared spectroscopy," Neuroimage 44(2), 428-447 (2009).

45. A. Rose, "Vision: human and electronic," Opt. Phys. Eng. 15, 197 (1973).

46. J. Xu et al., "FC-NIRS: a functional connectivity analysis tool for nearinfrared spectroscopy data," Biomed Res. Int. 2015, 100875 (2015).

47. T. J. Huppert et al., "HomER: a review of time-series analysis methods for near-infrared spectroscopy of the brain," Appl. Opt. 48(10), D280 (2009).

48. F. Scholkmann et al., "How to detect and reduce movement artifacts in near-infrared imaging using moving standard deviation and spline interpolation," Physiol. Meas. 31(5), 649-662 (2010).

49. P. L. Pan et al., "Aberrant spontaneous low-frequency brain activity in amnestic mild cognitive impairment: a meta-analysis of resting-state fMRI studies," Ageing Res. Rev. 35, 12-21 (2017).

50. S. L. Novi, R. B. M. L. Rodrigues, and R. C. Mesquita, "Resting state connectivity patterns with near-infrared spectroscopy data of the whole head," Biomed. Opt. Express 7(7), 2524 (2016).

51. M. A. Franceschini et al., "Diffuse optical imaging of the whole head," J. Biomed. Opt. 11(5), 054007 (2006).

52. F. Carbonell, P. Bellec, and A. Shmuel, "Global and system-specific resting-state fMRI fluctuations are uncorrelated: principal component analysis reveals anti-correlated networks," Brain Connect. 1(6), 496510 (2011).

53. E. J. Forero et al., "Use of near-infrared spectroscopy to probe occlusion severity in patients diagnosed with carotid atherosclerotic disease," Med. Res. Arch. 5(6), 1-22 (2017).

54. C. M. Lu et al., "Use of fNIRS to assess resting state functional connectivity," J. Neurosci. Methods 186(2), 242-249 (2010).

55. Y. He et al., "Optical mapping of brain activation during the English to Chinese and Chinese to English sight translation," Biomed. Opt. Express 8(12), 5399 (2017).

56. X. P. Ding et al., "Neural correlates of second-order verbal deception: a functional near-infrared spectroscopy (fNIRS) study," Neuroimage 87(9), 505-514 (2014).

57. C. Zich et al., "Simultaneous EEG-fNIRS reveals how age and feedback affect motor imagery signatures," Neurobiol. Aging 49, 183-197 (2017).
58. S. Montero-Hernandez et al., "Estimating functional connectivity symmetry between oxy- and deoxy-haemoglobin: implications for fNIRS connectivity analysis," Algorithms 11(5), 1-16 (2018).

59. G. Lee et al., "Cross-correlation between $\mathrm{HbO}$ and $\mathrm{HbR}$ as an effective feature of motion artifact in fNIRS signal," in 2018 6th Int. Conf. BrainComputer Interface (BCI 2018), pp. 1-3 (2018).

60. X. Cui, S. Bray, and A. L. Reiss, "Functional near infrared spectroscopy (NIRS) signal improvement based on negative correlation between oxygenated and deoxygenated hemoglobin dynamics," Neuroimage 49(4), 3039-3046 (2010).

61. J. Wang et al., "Corrigendum: GRETNA: a graph theoretical network analysis toolbox for imaging connectomics," Front. Hum. Neurosci. 9(June), 1-16 (2015).

62. M. Rubinov and O. Sporns, "Complex network measures of brain connectivity: uses and interpretations," Neuroimage 52(3), 1059-1069 (2010).

63. H. F. H. Ieong and Z. Yuan, "Abnormal resting-state functional connectivity in the orbitofrontal cortex of heroin users and its relationship with anxiety: a pilot fNIRS study," Sci. Rep. 7(March), 46522 (2017).

64. D. J. Watts and S. H. Strogatz, "Collective dynamics of 'small-world' networks," Nature 393(6684), 440-442 (1998).

65. Z. Wang et al., "Changes in the brain intrinsic organization in both on-task state and post-task resting state," Neuroimage 62(1), 394-407 (2012).

66. H. Niu et al., "Revealing topological organization of human brain functional networks with resting-state functional near infrared spectroscopy," PLoS One 7(9), 1-14 (2012).

67. J. Zhang et al., "Disrupted brain connectivity networks in drug-naive, first-episode major depressive disorder," Biol. Psychiatry 70(4), 334342 (2011).

68. X. Cui, "xjView: a viewing program for SPM," xjview 9.6 released, http://www.alivelearn.net/xjview (12 October 2017).

69. M. Xia, J. Wang, and Y. He, "BrainNet viewer: a network visualization tool for human brain connectomics," PLoS One 8(7), e68910 (2013).

70. M. Xia, "BrainNet Viewer," BrainNet Viewer Manual 1.61, https://www .nitrc.org/projects/bnv/ (7 January 2018).

71. F. McNab et al., "Common and unique components of inhibition and working memory: An fMRI, within-subjects investigation," Neuropsychologia 46(11), 2668-2682 (2008).

72. M. J. Herrmann et al., "Optical topography during a go-no go task assessed with multi-channel near-infrared spectroscopy," Behav. Brain Res. 160(1), 135-140 (2005).

73. A. K. Barbey, R. Colom, and J. Grafman, "Dorsolateral prefrontal contributions to human intelligence," Neuropsychologia 51(7), 1361-1369 (2013).

74. S. C. Andrews et al., "Improving working memory: The effect of combining cognitive activity and anodal transcranial direct current stimulation to the left dorsolateral prefrontal cortex," Brain Stimul. 4(2), 84-89 (2011).

75. S. Monsell, "Task switching," Trends Cogn. Sci. 7(3), 134-140 (2003).

76. Q. Zou et al., "Intrinsic resting-state activity predicts working memory brain activation and behavioral performance," Hum. Brain Mapp. 34(12), 3204-3215 (2013).

Zhishan $\mathrm{Hu}$ is a $\mathrm{PhD}$ candidate in neuroscience at the University of Macau, China. He uses functional near-infrared spectroscopy to investigate the neural basis underlying multiple cognitive processes.

Juan Zhang is an assistant professor at the University of Macau. She uses behavioral and neuroscience methodologies to investigate the brain mechanism underlying children's language and cognitive processes.

Lingyan Zhang is a radiologist at the Third Affiliated Hospital of China Southern Medical University. She is an expert in diagnosing and treating injuries and diseases using multimodal imaging techniques.

Yu-Tao Xiang is a full professor at the University of Macau. His research focuses on mood disorders, psychosis, psychopharmacology, and meta-analysis. $\mathrm{He}$ has received over 10 international research awards for his work.

Zhen Yuan is an associate professor at the University of Macau. His research focuses on neurosciences, neuroimaging, biomedical optics, and optical molecular imaging. 